\title{
Hepatic Stellate Cell Selective Disruption of Dynamin-2 GTPase Increases Murine Fibrogenesis through Up-Regulation of Sphingosine-1 Phosphate-Induced Cell Migration
}

Ruisi Wang, ${ }^{*}$ Qian Ding, ${ }^{\dagger}$ Thiago M. De Assuncao, ${ }^{\dagger}$ Taofic Mounajjed, ${ }^{\dagger}$ Jessica L. Maiers, ${ }^{\dagger}$ Changwei Dou, ${ }^{\dagger}$ Sheng Cao, ${ }^{\dagger}$ Usman Yaqoob, ${ }^{\dagger}$ Robert C. Huebert, ${ }^{\dagger}$ and Vijay H. Shah ${ }^{\dagger}$

From the Departments of Molecular Pharmacology and Experimental Therapeutics* and Gastroenterology and Hepatology, ${ }^{\dagger}$ and the Laboratory of Anatomic Pathology, ${ }^{\ddagger}$ Mayo Clinic, Rochester, Minnesota

Accepted for publication

September 1, 2016.

Address correspondence to Vijay H. Shah, M.D., Mayo Clinic, 200 First St SW,

Rochester, MN 55905. E-mail: shah.vijay@mayo.edu.

\begin{abstract}
Dynamin-2 (Dyn2) is implicated in endocytosis of receptor tyrosine kinases, which contribute to hepatic stellate cell (HSC) activation and liver fibrosis. A point mutation converting lysine 44 of Dyn2 to alanine (Dyn2K44A) disrupts its GTPase activity. We hypothesized that Dyn2K44A expression in HSCs would decrease HSC activation and fibrogenesis in vivo by disrupting receptor tyrosine kinase endocytosis and signaling. Dyn2K44A $A^{f l f l}$ mice were crossed with Collagen1-Cre $\left(\mathrm{Col1}^{\text {Cre }}\right)$ mice to generate offspring with HSC selective expression of Dyn2K44A (Col1 ${ }^{\text {Cre }} /$ Dyn2K44A $A^{f l f l}$ ). Contrary to our hypothesis, Col1 ${ }^{\text {Cre }}$ / Dyn2K44Aflfl mice showed increased hepatic fibrosis in response to liver injury. To elucidate mechanisms, we conducted in vitro experiments with HSCs infected with adenoviral vectors encoding LacZ, Dyn2K44A, or Dyn2WT. HSC-expressing Dyn2K44A displayed increased mRNA and protein levels of sphingosine kinase-1 (SK1), an enzyme previously implicated in the pathogenesis of fibrosis. To study the functional effects of Dyn2K44A regulation of SK1, we examined effects of AKT signaling and migration in HSCs. Dyn2K44A promoted both AKT phosphorylation and HSC migration in an SK1dependent manner. Genetic disruption of Dyn2 GTPase activity selectively in HSC enhances fibrogenesis, driven at least in part through up-regulation of the SK1 pathway and cell migration in HSCs. (Am J Pathol 2017, 187: 134-145; http://dx.doi.org/10.1016/j.ajpath.2016.09.001)
\end{abstract}

Liver fibrosis results from chronic damage to the liver in conjunction with the accumulation of extracellular matrix proteins. ${ }^{1}$ A dominant mechanism of fibrogenesis is the activation of hepatic stellate cells (HSCs), which differentiate into a myofibroblastic phenotype. ${ }^{1,2}$ Active myofibroblast-like cells are characterized by increased migration, $\alpha$-smooth muscle actin ( $\alpha$-SMA) expression, and robust collagen production. ${ }^{1,3}$ Despite the importance of HSC activation in fibrogenesis, there are no current therapies to effectively treat liver fibrosis. Further study is required to deepen our understanding of fibrogenesis mechanisms to successfully treat hepatic fibrosis. ${ }^{2}$

Dynamins are highly conserved large GTPases involved in a wide range of cellular functions. Their most recognized function is in endocytosis, mediating the GTPase-dependent fission of vesicles generated from the plasma membrane by clathrin-dependent and clathrin-independent mechanisms. ${ }^{4}$ The dynamin family is composed of three members: dynamin-1, dynamin-2 (Dyn2), and dynamin-3. Dyn2 is ubiquitously expressed and regulates both receptormediated endocytosis and vesicle transport between endomembrane system compartments. ${ }^{4-8}$ A point mutation

Supported by NIH grants DK59615 and AA021171 (V.H.S.) and T32 DK07198 (J.L.M.), the Clinical Core of the Mayo Clinic Center for Cell Signaling in Gastroenterology grant P30DK084567, and American Liver Foundation Postdoctoral Fellowship (J.L.M.).

Disclosures: None declared. 
converting lysine 44 of Dyn2 to alanine (Dyn2K44A) disrupts GTPase activity and is commonly used to experimentally probe dynamin endocytic functions. ${ }^{1,9}$ Prior studies have indicated that loss of Dyn2 impairs integrin internalization in endothelial cells, increasing cell surface levels of integrins, which led to impaired angiogenesis. ${ }^{2,8}$ Other work also showed that endothelial cell (EC)specific overexpression of Dyn2K44A impaired angiogenesis in vivo through disrupting receptor tyrosine kinase (RTK) function. ${ }^{1,2,10}$ RTKs play an important role in regulating HSC function and liver fibrosis; however, direct evidence that Dyn2 regulates HSC function and liver fibrosis in vivo is lacking. ${ }^{1,3,11}$ Based on these results, we hypothesized that disruption of Dyn2 function in HSCs through expression of Dyn2K44A might also disrupt tyrosine kinase-dependent HSC activation and fibrogenesis in vivo.

In the present study, we sought to identify the effect of Dyn2K44A on liver fibrosis and the intrinsic mechanisms that regulate this effect. We established and used transgenic mice selectively overexpressing the dominant negative Dyn2K44A in HSCs by crossing Dyn $2 K 44 A^{\text {flfl }}$ with collagen 1-Cre $\left(\mathrm{Coll}^{\mathrm{Cre}}\right)$. These mice were used to examine the in vivo role of Dyn2 GTPase function in fibrogenesis. Contrary to our hypothesis, Coll ${ }^{\mathrm{Cre}} / \mathrm{Dyn} 2 K 44 A^{\text {flffl }}$ mice displayed exacerbated fibrosis in both bile duct ligation (BDL) and carbon tetrachloride models. These observations led us to investigate how Dyn2 inhibition exacerbates fibrogenesis. HSCs were engineered to overexpress Dyn2K44A using an adenoviral system. HSCs expressing Dyn2K44A displayed increased mRNA and protein levels of sphingosine kinase-1 (SK1), an enzyme previously implicated in the pathogenesis of fibrosis in part through effects on HSC migration. ${ }^{12}$ Dyn2K44A increased AKT phosphorylation in HSCs in an SK1-dependent manner. Conditioned media from Dyn2K44A-infected cells induced HSC migration in an SK1- and AKT-dependent manner. In total, these studies uncover a new role for Dyn2K44A in fibrogenesis and elucidate mechanisms of Dyn2K44A in the enhanced activation of HSCs.

\section{Materials and Methods}

\section{Generation of Col1 ${ }^{\text {Cre }} /$ DynK $44 A^{f l f l}$ Mice}

$\operatorname{Dyn} 2 K 44 A^{f l f l}$ mice were generated and genotyped, as previously described, ${ }^{1,3}$ and were crossed with Coll $^{\text {Cre }}$ mice $^{4,13}$ to generate offspring with HSC-selective expression of DynK44A $\left(\right.$ Coll $^{\text {Cre }} /$ Dyn $\left.2 K 44 A^{f / A}\right)$. All of the offspring were screened and genotyped by PCR for the presence of Coll $^{\text {Cre }}$ and Dyn2K44A Alff . Because Dyn2K44A is a dominant negative mutant, single or double copy of $\mathrm{Coll}^{\mathrm{Cre}}$ and Dyn2K44A $A^{f l / f}$ is sufficient for Dyn2K44A overexpression in HSCs. HSC isolation was performed to confirm the overexpression of Dyn2K44A in HSCs. The Mayo Clinic
(Rochester, MN) Institutional Animal Care and Use Committee approved all animal studies.

\section{Liver Cell Isolation}

\section{HSC Isolation and Culture}

HSCs were isolated from $\mathrm{Coll}^{\text {Cre }} / \mathrm{Dyn} 2 \mathrm{~K} 44 A^{\text {flffl }}$ mice and littermate controls, as described previously. ${ }^{6-8}$ Briefly, after in situ perfusion of the liver with pronase (Roche, Indianapolis, IN), followed by collagenase (Roche), dispersed cell suspensions were layered on a discontinuous density gradient of $15.6 \%$ Accudenz (Accurate Chemical and Scientific, Westbury, NY). The resulting upper layer consisted of $>95 \%$ stellate cells. The cell viability was verified by phase-contrast microscopy as well as the ability to exclude propidium iodide. The viability of all cell cultures used for the study was $>95 \%$.

\section{Hepatocyte Isolation}

Hepatocytes were isolated from $C_{0 l 1}^{C r e} / D y n 2 K 44 A^{f / f l}$ mice and littermate controls, as described previously. ${ }^{14,15}$ Briefly, a two-step collagenase perfusion technique was performed. After in situ perfusion of the liver with a calcium-free medium, followed by collagenase (Roche), dispersed cell suspensions were purified by Percoll solution (Sigma, St. Louis, MO). The hepatocytes were cultured in Dulbecco's modified Eagle's medium containing $10 \%$ fetal bovine serum and $1 \%$ penicillin/ streptomycin (Gibco, Waltham, MA). ${ }^{2,8,16}$

\section{EC Isolation}

ECs were isolated from Coll $^{\text {Cre }} / D y n 2 K 44 A^{f / f l}$ mice and littermate controls, as described previously. ${ }^{12}$ Briefly, liver tissue was perfused, harvested, dissected, minced, and digested in a collagenase buffer. The tissue was then incubated with immunomagnetic Dynabeads (Dynal, Waltham, MA) coated with rat anti-mouse CD146 (BD Biosciences, Franklin Lakes, NJ), an endothelial marker, for 1 hour at room temperature. Cells were separated with a magnet and plated on collagen I-coated dishes. Viability was $>90 \%$ by trypan blue staining, and purity was $>95 \%$ by staining for CD31. Cells were grown in EC growth medium containing $5 \%$ fetal bovine serum, $2 \%$ EC growth supplement, and $1 \%$ penicillin/streptomycin (ScienCell, San Diego, CA) and maintained in standard tissue culture conditions $\left(37^{\circ} \mathrm{C}, 5 \%\right.$ $\mathrm{CO}_{2}$ incubator).

\section{Viral Transfection of Cells}

Cell lines were used for in vitro viral transfection. LX-2 (immortalized human HSC) cells and immortalized liver sinusoidal ECs were grown in Dulbecco's modified Eagle's medium containing $10 \%$ fetal bovine serum and $1 \%$ penicillin/streptomycin (Gibco). ${ }^{16}$ Liver hepatocellular carcinoma cells (HepG2) were routinely maintained in Dulbecco's modified Eagle's medium, supplemented with $10 \%$ fetal bovine serum and $1 \%$ penicillin/streptomycin. 
Adenoviral vectors were generated through the Iowa Vector Core and encoded Dyn2 K44A or LacZ control and Dyn2WT. Cells were incubated for 12 hours with $0.1 \%$ albumin/phosphate-buffered saline with 50 multiplicity of infection of adenoviruses, which achieved transduction efficiency approximating $90 \%$ with minimal toxicity. All cell lines were maintained in standard tissue culture conditions $\left(37^{\circ} \mathrm{C}, 5 \% \mathrm{CO}_{2}\right.$ incubator).

\section{Biotinylation Assay for Plasma Membrane Receptor Level}

Biotin-based assays were performed as described previously. ${ }^{13}$ HSCs were starved overnight. Subsequently, the cells were placed on ice and washed once with cold Dulbecco's phosphate-buffered saline. Cell surface proteins were labeled with $1 \mathrm{mg} / \mathrm{mL}$ of EZ-link cleavable sulfoNHS-SS-biotin (21331; Thermo Scientific, Waltham, MA) in Dulbecco's phosphate-buffered saline (14040; Invitrogen, Waltham, MA) for 15 minutes at $4^{\circ} \mathrm{C}$. Cells were lysed by scraping in radioimmunoprecipitation assay lysis buffer with protease inhibitor cocktails (Roche) and incubation at $4^{\circ} \mathrm{C}$ for 20 minutes. Cell extracts were cleared by centrifugation $\left(15,000 \times g, 10\right.$ minutes, $\left.4^{\circ} \mathrm{C}\right)$. Cell lysates were subjected to protein $\mathrm{G}$ sepharose beads (17-0618-01; GE Health Care, Fairfield, CT) pull down, followed by Western blot (WB) for transferrin receptor (TfR). Integrated densities of the protein bands were quantified by ImageJ version $1.51 \mathrm{f}(\mathrm{NIH}$, Bethesda, MD).

\section{Induction of Liver Fibrosis in Vivo}

Two models of cirrhosis were used: BDL and chronic administration of carbon tetrachloride. BDL or a sham operation was performed, as previously described, to induce fibrosis. ${ }^{4,17,18}$ For chronic carbon tetrachloride administration, mice were subjected to $\mathrm{CCl}_{4}(1 \mu \mathrm{L} / \mathrm{g})$ or olive oil injection i.p. twice a week for 6 weeks, as described previously. ${ }^{13}$ At the time of sacrifice, whole livers and blood were collected for analysis from $\mathrm{Coll}^{\mathrm{Cre}} / \mathrm{Dyn} 2 \mathrm{~K} 44 \mathrm{~A}^{\text {fl/f }}$ mice and littermate controls. All surgeries were performed using appropriate anesthesia, analgesia, and antibiotics.

\section{Serum Analysis}

Whole blood was isolated from all mice, kept at room temperature for 2 hours, and then centrifuged at $4000 \times g$ for 8 minutes to collect serum. Serum was then analyzed as previously described (North Carolina Animal Lab Test Center, Chapel Hill, NC). ${ }^{13}$

\section{Sirius Red Staining}

Livers samples were fixed in $10 \%$ phosphate-buffered formalin and embedded in paraffin. Sections $(5 \mu \mathrm{m}$ thick) were stained with picrosirius red (Sigma) and counterstained with fast green (Sigma). The proportion of tissue stained with picrosirius red content was quantified with ImageJ.

\section{Immunofluorescence}

$\alpha$-SMA and fibronectin (FN) immunofluorescence staining were performed using frozen sections. Embedded liver blocks were divided into sections ( $5 \mu \mathrm{m}$ thick) and fixed in ice-cold acetone or methanol for 10 minutes. Samples were blocked with $10 \%$ goat serum in phosphate-buffered saline for 1 hour and then blocked with mouse IgG (MKB-2213) for 1 hour. Samples were incubated with mouse $\alpha$-SMA (Sigma A5228, 1:1000) or mouse anti-FN (BD Biosciences $610077,1: 500$ ) overnight at $4{ }^{\circ} \mathrm{C}$, followed by incubation for 1 hour with Alexa Fluor 488-conjugated goat anti-mouse (1:250) secondary antibody. The slides were then counterstained with TOTO3, and confocal microscopy was performed by LSM 5 Pascal (Zeiss, Oberkochen, Germany), in which appropriate laser and filter combinations were selected according to excitation and emission spectrum features of the Alexa fluorochromes. Fluorescence was quantitated in randomly chosen sections and quantified by ImageJ $(\times 10$ magnification; 10 fields each from each sample).

\section{Real-Time PCR}

An RNeasy kit (Qiagen, Hilden, Germany) was used to extract total RNA from cells and mouse tissue, according to the manufacturer's instructions. mRNA $(10 \mu \mathrm{g})$ was used for cDNA synthesis with dNTP and oligo primer using SuperScript III (Invitrogen) first-strand synthesis system for real-time PCR, per the manufacturer's protocol. Real-time PCR was performed with the same amount of cDNA in a total reaction using IQ SYBR Green Mix (Biorad, Hercules, CA) and the 7500 Real-Time PCR system (Applied Biosystems), according to the manufacturer's instructions.

\section{Table 1 Primer Sequences}

\begin{tabular}{|c|c|c|}
\hline Primer & Sequence & Species \\
\hline \multirow[t]{2}{*}{ GAPDH } & F: 5'-CCAGGGCTGCTTTTAACTCT-3' & Human \\
\hline & R: 5'-GGACTCCACGACGTACTCA-3' & \\
\hline \multirow[t]{2}{*}{$\alpha-S M A$} & F: $5^{\prime}$-GGAGATCACGGCCCTAGCAC-3' & Human \\
\hline & R: 5'-AGGCCCGGCTTCATCGTAT-3' & \\
\hline \multirow[t]{2}{*}{ Fibronectin } & F: 5'-GATAAATCAACAGTGGGAGC-3' & Human \\
\hline & R: 5'-CCCAGATCATGGAGTCTTTA-3' & \\
\hline \multirow[t]{2}{*}{ ColI } & F: 5'-TGTGAGGCCACGCATGAG-3' & Human \\
\hline & R: 5'-CAGATCACGTCATCGCACAA-3' & \\
\hline \multirow[t]{2}{*}{$\beta$-actin } & F: 5'-AGAGGGAAATCGTGCGTGAC-3' & Mouse \\
\hline & R: 5'-CAATAGTGATGACCTGGCCGT-3' & \\
\hline \multirow[t]{2}{*}{$\alpha-S M A$} & F: $5^{\prime}-$ AAACAGGAATACGACGAAG- $3^{\prime}$ & Mouse \\
\hline & R: 5'-CAGGAATGATTTGGAAAGGA-3' & \\
\hline \multirow[t]{2}{*}{ Fibronectin } & F: 5'-GTGGCTGCCTTCAACTTCTC-3' & Mouse \\
\hline & R: 5'-GTGGGTTGCAAACCTTCAAT-3' & \\
\hline
\end{tabular}

Col, collagen; F, forward; GAPDH, glyceraldehyde-3-phosphate dehydrogenase; $R$, reverse; $\alpha$-SMA, $\alpha$-smooth muscle actin. 

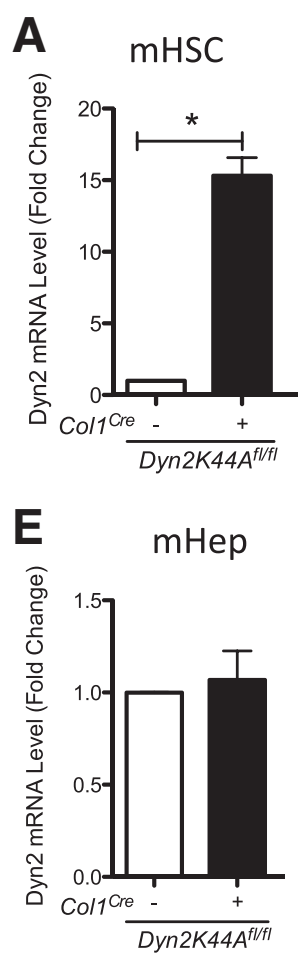

B $\quad \mathrm{mHSC}$

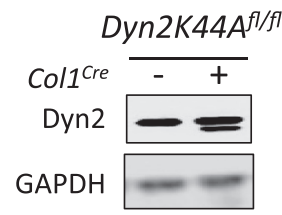

$\mathbf{F}$

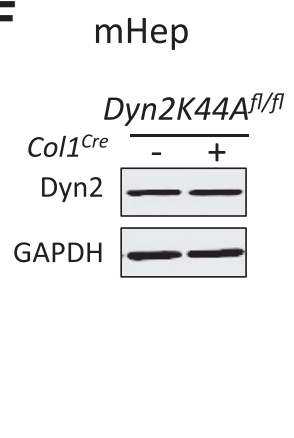

C $\quad \mathrm{mEC}$

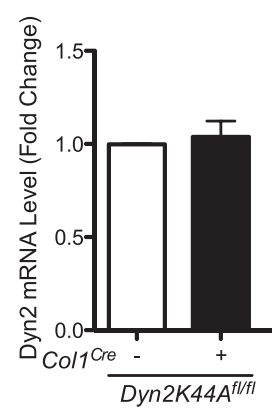

G

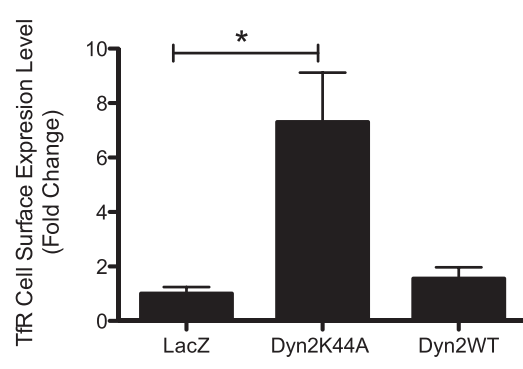

mEC

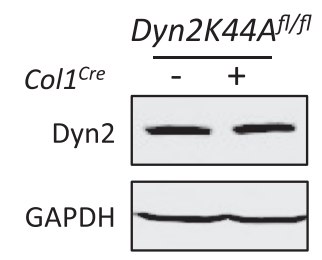

Figure 1 Characterization of Col1 ${ }^{\text {Cre }} / D y n 2 K 44 A^{f l f f l}$ mice. A: mRNA from isolated HSCs from Col1 ${ }^{\text {Cre }} /$ Dyn2K44A $A^{f l f l}$ mice and littermate controls was subjected to real-time PCR for Dyn2 quantification. B: Primary HSC lysates were isolated and subjected to Western blot (WB). Glyceraldehyde-3-phosphate dehydrogenase (GAPDH) served as a loading control. The higher band represented endogenous Dyn2, and the lower band represented the overexpression of rat Dyn2 construct. C-F: mRNA and protein from isolated endothelial cells (ECS) and hepatocytes (Hep) from Col1 ${ }^{\text {Cre }} /$ Dyn2K $44 A^{f l / f l}$ mice and littermate controls were subjected to real-time PCR and WB for Dyn2 quantification (no significant change between groups). G: HSCs were infected by LacZ, Dyn2K44A, and Dyn2WT adenovirus. Infected HSCs were subjected to biotinylation assay to examine transferrin receptor (TfR) expression on the HSC surface, followed by streptavidin pull down and WB analysis for TfR. Whole cell lysate served as a loading control. $n=3(\mathbf{A}) ; n=4$ (C and E); $n=5$ (G). ${ }^{*} P<0.05$. WT, wild-type.

Amplification of glyceraldehyde-3-phosphate dehydrogenase and $\beta$-actin was performed in the same reaction for respective samples as internal control. Each experiment was performed in triplicate. Primer sequences are listed in Table 1.

\section{Western Blot}

Cells or liver tissue was lysed and prepared for WB analysis, as previously described. ${ }^{19}$ Immunoblot was performed according to the protocol recommended for individual antibodies, Dyn2 (gift from Dr. Mark A. McNiven, Ph.D., Rochester, MN), $\alpha$-SMA (A5228; Sigma), FN (SC9068; Santa Cruz, Santa Cruz, CA), Col I (1310-01; SouthernBiotech, Birmingham, AL), glyceraldehyde-3-phosphate dehydrogenase (Am4300; Ambion, Waltham, MA), pAKT (9271; Cell Signal, Danvers, MA), and tAKT (9272S; Cell Signal). Immunoreactive bands were visualized using horseradish peroxidase-conjugated secondary antibody and the enhanced chemiluminescent system (Santa Cruz Biotechnology, Inc.). All experiments were done in triplicate, and quantitation was done by densitometry using ImageJ.

\section{Hydroxyproline Assay}

Hydroxyproline content in whole liver specimens was measured colorimetrically, as described. ${ }^{17,18}$ Animal liver samples were weighted and then acid hydrolyzed. Hydroxyproline concentration was determined from hydroxyproline (Sigma) standard curve and expressed as $\mu \mathrm{g}$ hydroxyproline/mg liver tissue.

\section{Transwell Migration Assay}

Conditioned media from LacZ, Dyn2K44A, or Dyn2WT transfected HSCs were used to assess HSC migration measured by transwell assay. ${ }^{13}$ Briefly, $8-\mu \mathrm{m}$-pore polycarbonate filters (3422; Costar, Boston, MA) were coated with $1 \%(\mathrm{w} / \mathrm{v})$ collagen (Sigma-Aldrich, St. Louis, MO). Cells $\left(1 \times 10^{4}\right.$ cells in $100 \mu \mathrm{L}$ per well $)$ were suspended in serum-free Dulbecco's modified Eagle's medium. Conditioned media or indicated compound was added to the lower chamber. After incubation at $37^{\circ} \mathrm{C}$ for 12 hours, migrated cells were stained by crystal violet. The number of cells that migrated to the bottom side of the chamber was determined by counting 
A

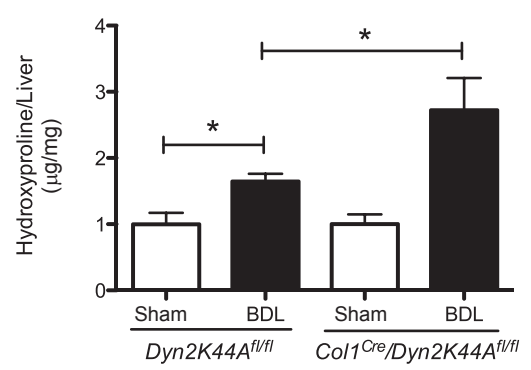

B

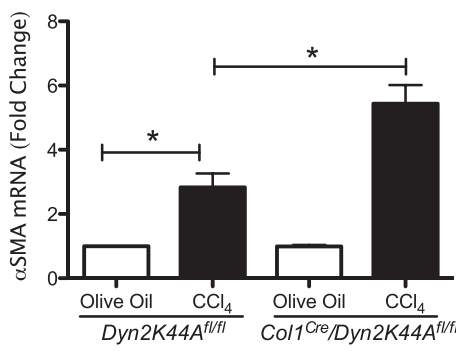

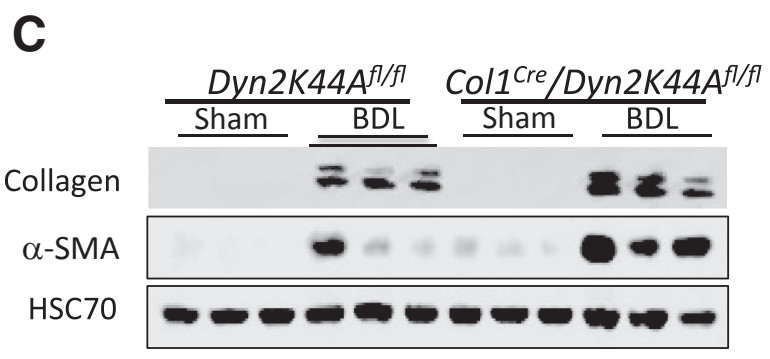

D

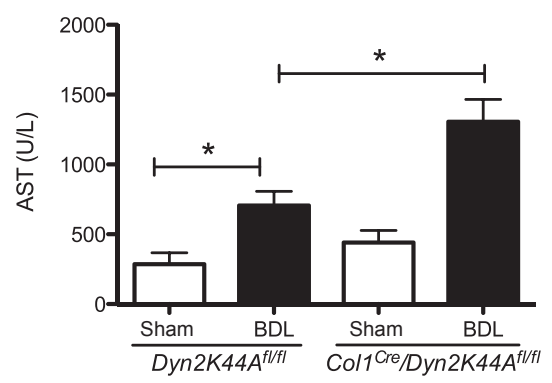

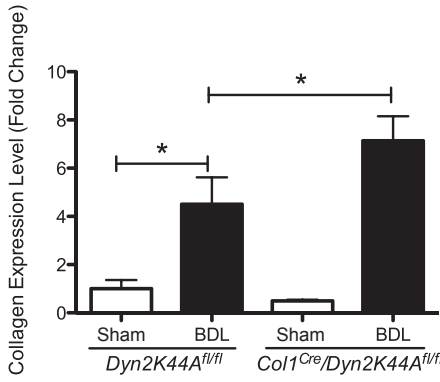
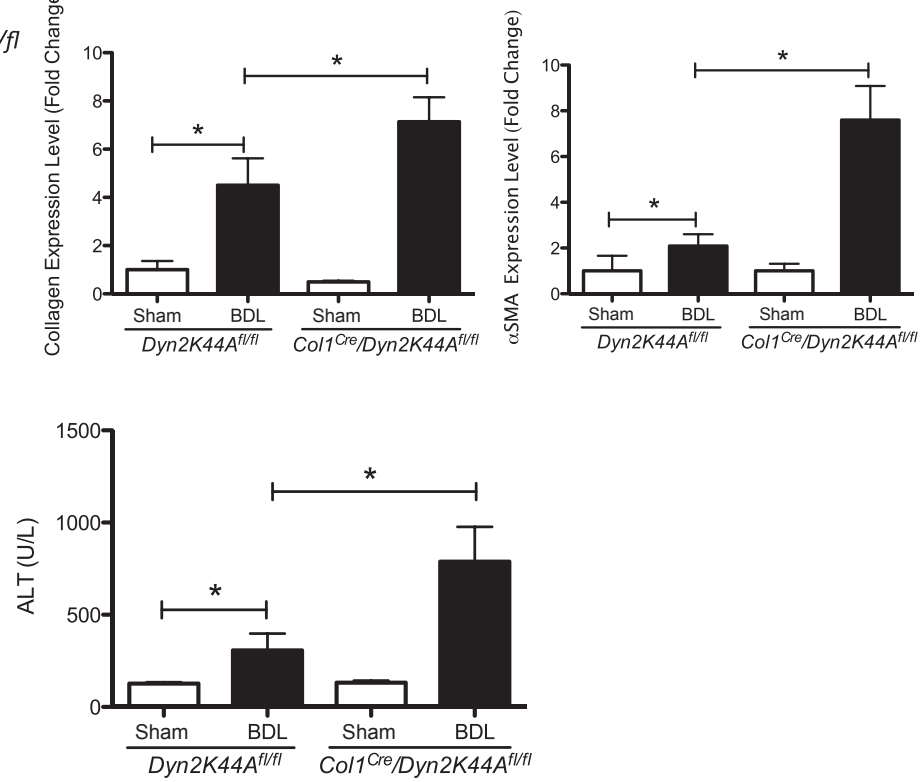

Figure $2 \mathrm{Col1}^{\mathrm{Cre}} / \mathrm{Dyn} 2 K 44 A^{f l f l}$ mice show enhanced liver fibrosis in response to bile duct ligation (BDL)-induced injury. Dyn2K44A $A^{f l f l}$ and $C o l 1^{\text {Cre }} /$ Dyn2K44A $A^{f l f l}$ mice were subjected to BDL or sham operation, and livers were harvested after 3 weeks. A: Liver samples were subjected to hydroxyproline assay to examine collagen content. B: Whole liver samples were subject to real-time PCR for $\alpha$-smooth muscle actin $(\alpha$-SMA) quantification. C: Liver samples from sham and BDL Dyn2K44A $A^{f l f l}$ and Col1 ${ }^{\text {Cre }} /$ Dyn2K44A $A^{f l f l}$ mice were subjected to Western blot to analyze $\alpha$-SMA and collagen I expression. D: Levels of aspartate aminotransferase (AST) and alanine aminotransferase (ALT) were measured by serum biochemical analysis in all four groups mice. $n=6\left(\right.$ A - D). ${ }^{*} P<0.05$. $\mathrm{CCl}_{4}$, carbon tetrachloride.

the number of cells per field by light microscopy ( $\times 10$ magnification).

\section{Transcriptional Reporter Assays}

The human SKI gene was amplified and transferred into the pGL3 basic vector, as described before. ${ }^{20}$ HSCs $\left(1 \times 10^{6}\right)$ were transfected using Effectene transfection reagent (Qiagen) with $400 \mathrm{ng}$ of reporter plasmid of the human SK1 gene or a control plasmid, along with a simultaneous infection of adenovirus for LacZ. Cells transfected with a renilla construct served as a positive control. After 48 hours of transfection and infection, cells were collected, and then relative luciferase units (RLUs) were measured using the luciferase assay system (Promega, Madison, WI) and a Turner 20/20 luminometer (Promega). Each experiment was performed in triplicate at a minimum. Renilla readings were measured and used for normalization in all experiments.

\section{Results}

\section{Characterization of HSC-Selective Expression of Dyn2K44A in Col1 ${ }^{\mathrm{Cre}} / \mathrm{Dyn} 2 \mathrm{~K} 44 \mathrm{~A}^{\mathrm{fl} / \mathrm{fl}}$ Mice}

To test our hypothesis that Dyn2K44A served as a modifier of the HSC response during chronic liver injury, we generated a transgenic mouse containing a floxed stop codon that preceded a Dyn2 gene containing a point mutation at DynK44A. ${ }^{10}$ These mice were crossed with a mouse line in which Cre expression is driven by the Col1. ${ }^{21}$ Because Coll is selectively expressed in HSCs within the liver, offspring overexpress Dyn2K44A selectively in HSCs. To confirm the overexpression of Dyn2K44A, HSCs were isolated and analyzed. Real-time PCR showed that the Dyn2 mRNA levels in HSCs from $\mathrm{Coll}^{\mathrm{Cre}} / \mathrm{Dyn} 2 \mathrm{~K} 44 \mathrm{~A}^{f l / f}$ mice were 15 times higher than those of littermate controls (Figure 1A). WB confirmed that HSCs from $\mathrm{Coll}^{\mathrm{Cre}} / \mathrm{Dyn} 2 \mathrm{~K} 44 \mathrm{~A}^{f l f l}$ mice expressed 


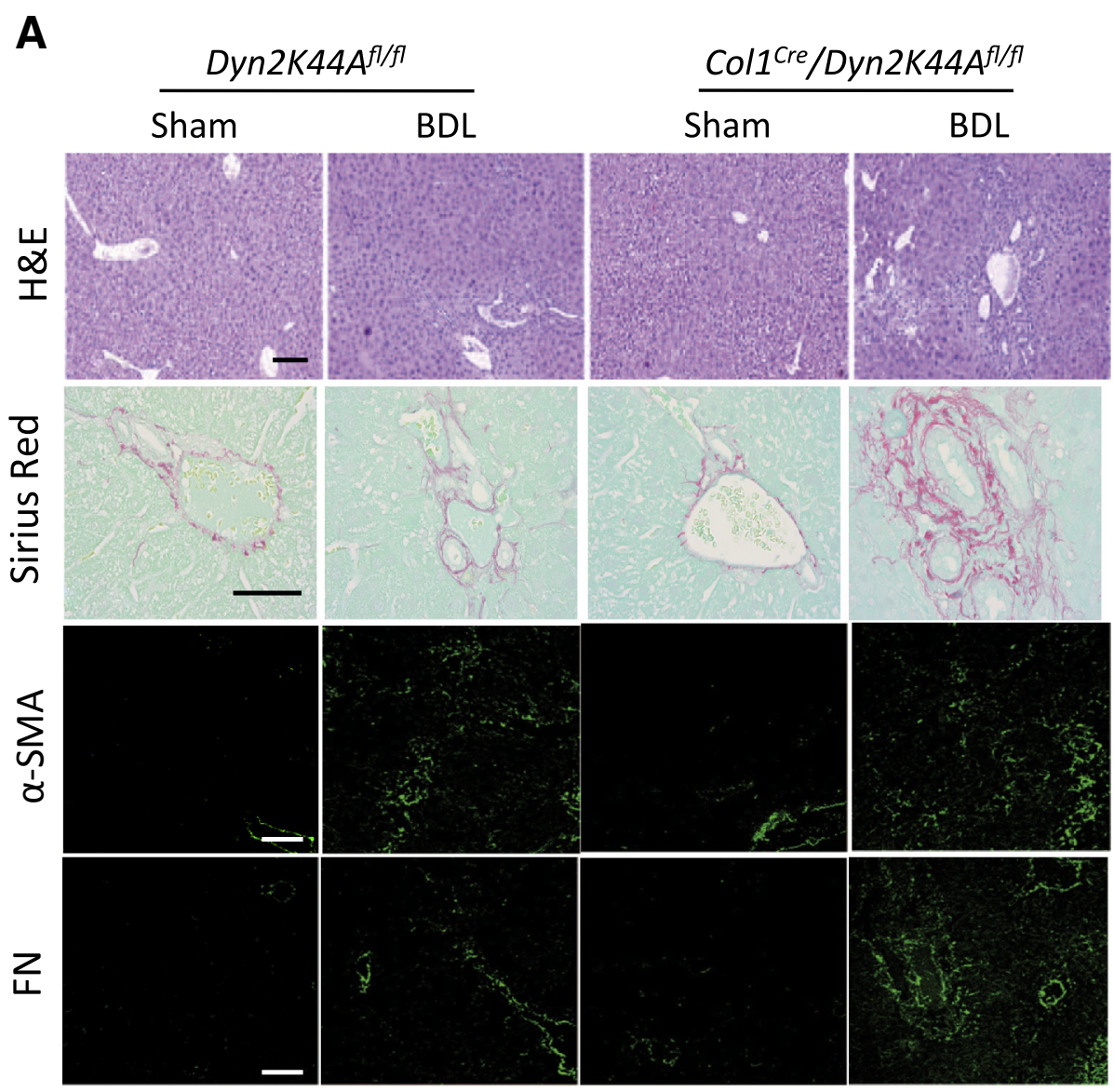

Figure $3 \quad \mathrm{Col}^{\mathrm{Cre}} / \mathrm{Dyn} 2 K 44 A^{\mathrm{fl} f l}$ mice display enhanced fibrosis in response to bile duct ligation (BDL)-induced injury. Mice were subjected to sham or BDL surgery for 3 weeks. A: Hematoxylin and eosin (H\&E) staining and Sirius Red staining were performed from paraffin liver sections. Immunofluorescence was performed on frozen liver sections using antibodies against $\alpha$-smooth muscle actin ( $\alpha$-SMA; green) and fibronectin (FN; green). B-D: Collagen deposition, $\alpha$-SMA, and FN were quantified using ImageJ, as detailed in Materials and Methods. $n=6$ (B-D). ${ }^{*} P<0.05$. Scale bars $=50 \mu \mathrm{m}$.

B

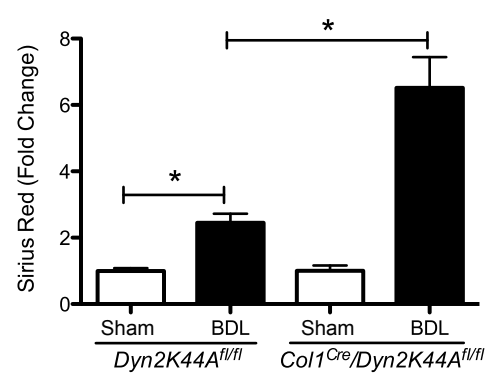

C

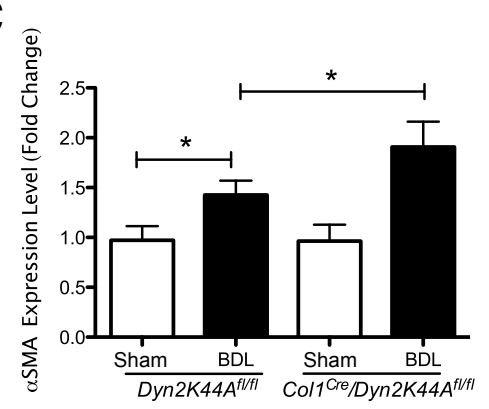

D

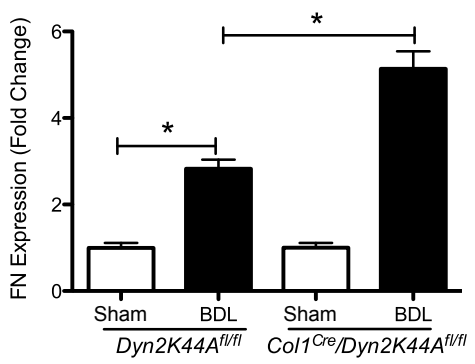

Dyn2K44A, as evidenced by greater Dyn2 antibody immunoreactivity compared to littermate controls (Figure 1B). We confirmed the specificity of Dyn2K44A expression in HSCs by isolating primary hepatocytes or ECs from mice and analyzing Dyn2 expression using realtime PCR and WB (Figure 1, C-F). The endocytic pathway involves internalization of both clathrin- and caveolin-coated vesicles, both of which are mediated by Dyn2 GTPase activity. ${ }^{19}$ We therefore perturbed Dyn2 function and assessed whether receptor endocytosis was affected. HSCs were infected with the Dyn2K44A adenovirus or adenovirus encoding LacZ or Dyn2WT as controls. TfR was used as a prototypical receptor that undergoes Dyn2-dependent endocytosis, and surface levels were analyzed by biotin labeling assay. ${ }^{22}$ Dyn2K44A cells displayed increased TfR on the cell surface, with no change in TfR expression in the total cell lysate (Figure 1G). These data provide evidence that Dyn2K44A was inhibiting canonical dynamin functions in HSCs. These data validated that $\mathrm{Col1}^{\mathrm{Cre}} / \mathrm{Dyn} 2 \mathrm{~K} 44 A^{f / f l}$ mice provided an appropriate model to explore Dyn2K44A function selectively in HSCs.

\section{BDL- or Carbon Tetrachloride-Induced Liver Injury Leads to Enhanced Fibrosis in Col1 ${ }^{\text {re }} /$ Dyn2K44A $A^{f l f l}$ Mice}

Coll ${ }^{\text {Cre }} /$ Dyn $2 K 44 A^{f / f l}$ mice and littermate controls were subjected to two different models of liver injury. First, BDL or sham surgeries were performed in Coll ${ }^{\text {Cre }} /$ Dyn $2 K 44 A^{\text {fl/fl }}$ 


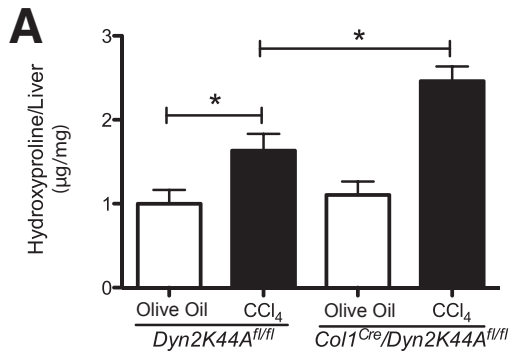

C

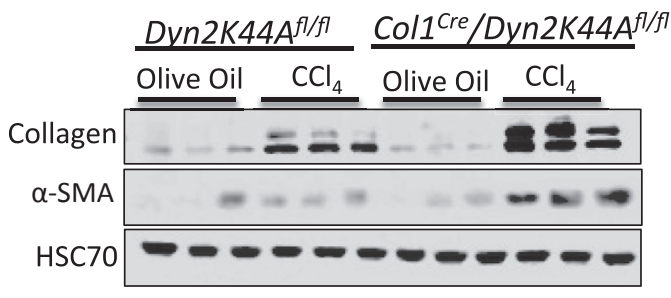

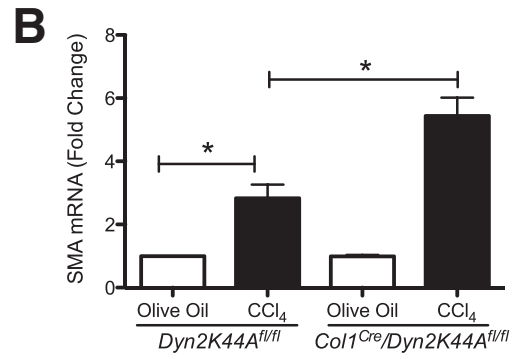

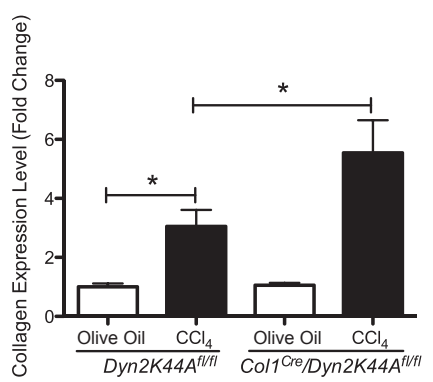

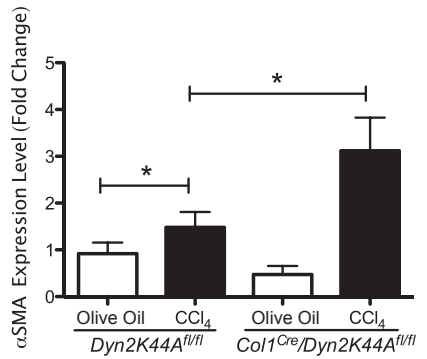

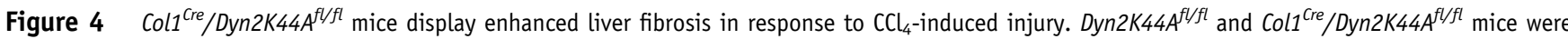
subjected to 6 weeks of $\mathrm{CCl}_{4}$ or olive oil treatment. A: Liver samples were subjected to hydroxyproline assay to examine collagen deposition. B: mRNA was harvested from whole liver samples and subjected to real-time PCR to examine $\alpha$-smooth muscle actin ( $\alpha$-SMA) quantification. C: Liver samples from olive oil or $\mathrm{CCl}_{4}$ treated from Dyn2K44A $A^{f l f l}$ and $\mathrm{Col}^{\text {Cre }} /$ Dyn2K44A $A^{f l f l}$ mice were subjected to Western blot for $\alpha-\mathrm{SMA}$ and collagen I quantification. $n=8(\mathbf{A}-\mathrm{C})$. ${ }^{*} P<0.05 . \mathrm{CCl}_{4}$, carbon tetrachloride.

mice or littermate controls. A hydroxyproline assay showed more liver collagen after BDL in $\mathrm{Coll}^{\mathrm{Cre}} / \mathrm{Dyn} 2 \mathrm{~K} 44 \mathrm{~A}^{\mathrm{fl} / \mathrm{fl}}$ compared to controls (Figure 2A). To further determine the effect of Dyn2K44A on liver fibrosis, mRNA from whole livers of these animals was isolated to evaluate the hepatic fibrotic marker $\alpha$-SMA. Expression of $\alpha$-SMA was higher in $\mathrm{Coll}^{\mathrm{Cre}} / \mathrm{Dyn} 2 \mathrm{~K} 44 \mathrm{~A}^{f l f l}$ mice after BDL compared to littermate controls (Figure 2B). To further characterize our Dyn2K44A model, we used WB to determine the protein level of $\alpha$-SMA and ColI. ColI and $\alpha$-SMA both increased significantly more after BDL in $\operatorname{Coll}^{\text {Cre }} /$ Dyn $2 K 44 A^{f / f l}$ compared to control (Figure 2C). Significantly higher levels of alanine aminotransferase and aspartate aminotransferase were also observed in $\mathrm{Coll}^{\mathrm{Cre}} / \mathrm{Dyn} 2 \mathrm{~K} 44 \mathrm{~A}^{f / / f}$ mice after BDL compared with littermate controls (Figure 2D). Tissue sections were used to examine the histopathological characterization between groups by hematoxylin and eosin, Sirius Red, and immunofluorescence staining for $\alpha$-SMA and FN (Figure 3A). Sirius Red staining revealed significantly higher collagen deposition after BDL in Coll $^{\text {Cre }} /$ Dyn2$K 44 A^{f l f l}$ mice compared with littermate control mice (Figure 3B). Immunofluorescence of $\alpha$-SMA and FN showed similar, statistically significant, increases in Coll${ }^{C r e} / D y n 2 K 44 A^{f l f l}$ mice compared to controls (Figure 3, $\mathrm{C}$ and $\mathrm{D})$. These in vivo data suggested that, in contrast to our original hypothesis, Dyn2K44A expression in HSCs led to more severe fibrogenesis in response to liver injury.

Next, we confirmed our findings using a second fibrosis model, carbon tetrachloride. Similar to that observed after BDL, hydroxyproline assay showed that livers from carbon tetrachloride-treated $\mathrm{Coll}^{\mathrm{Cre}} / \mathrm{Dyn} 2 \mathrm{~K} 44 \mathrm{~A}^{\mathrm{flfl}}$ mice contained more collagen than controls (Figure 4A). $\alpha$-SMA mRNA levels were also significantly higher in carbon tetrachloride-treated $\mathrm{Coll}^{\mathrm{Cre}} / \mathrm{Dyn} 2 \mathrm{~K} 44 \mathrm{~A}^{\mathrm{fl} / \mathrm{fl}}$ mice compared to littermate controls (Figure 4B). ColI and $\alpha$-SMA protein levels were also higher in $\mathrm{Coll}^{\mathrm{Cre}} / \mathrm{Dyn} 2 \mathrm{~K} 44 \mathrm{~A}^{\text {flff }}$ mice compared with littermate controls receiving carbon tetrachloride (Figure 4C). The histopathological characterization is shown by representative images in Figure 5A. Fibrosis was reviewed and scored via hematoxylin and eosin and Sirius Red staining by a blinded pathologist (T.M.), which indicated more fibrosis in carbon tetrachloride-treated Coll ${ }^{\text {Cre }} /$ Dyn $2 K 44 A^{f l f l}$ mice (Figure 5B). Collagen deposition was significantly higher in carbon tetrachloride-treated $\mathrm{Coll}^{\mathrm{Cre}} / \mathrm{Dyn} 2 \mathrm{~K} 44 \mathrm{~A}^{\mathrm{fl} / \mathrm{fl}}$ mice compared with carbon tetrachloride-treated littermate control mice, as examined by Sirius Red staining (Figure 5C). In addition, $\alpha$-SMA and FN immunofluorescence staining (Figure 5, D and E) showed significant increases in Dyn2K44A mice compared to controls. Together, both carbon tetrachloride and BDL models revealed that Dyn2K44A expression in HSCs led to more severe fibrogenesis in response to liver injury.

\section{Disruption of Dyn2 Activity Enhances HSC SK1 Expression}

Our finding that Dyn2K44A expression in HSCs sensitized mice to fibrogenesis suggested that Dyn2 inhibition promoted HSC activation. Previous studies from our laboratory 


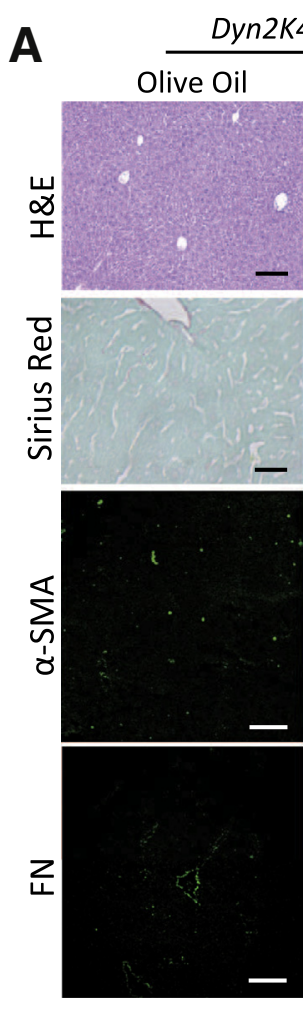

B

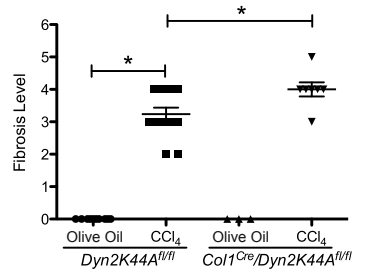

$\mathrm{CCl}_{4}$
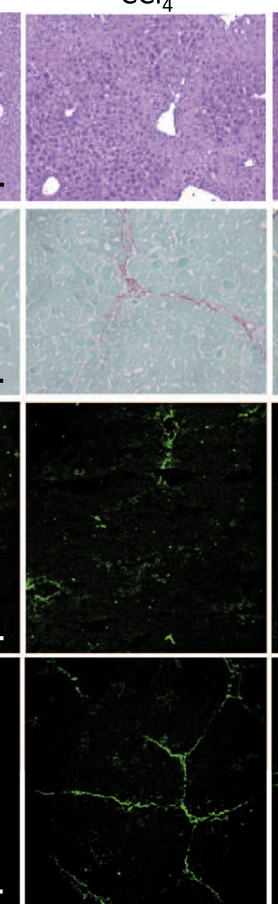

C
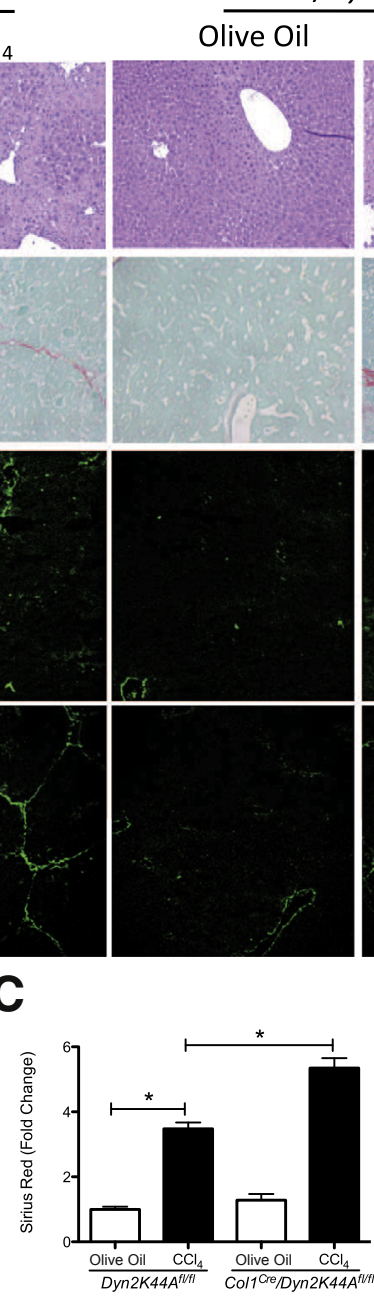

Col1 ${ }^{\text {Cre }} /$ Dyn2K44A f/ff
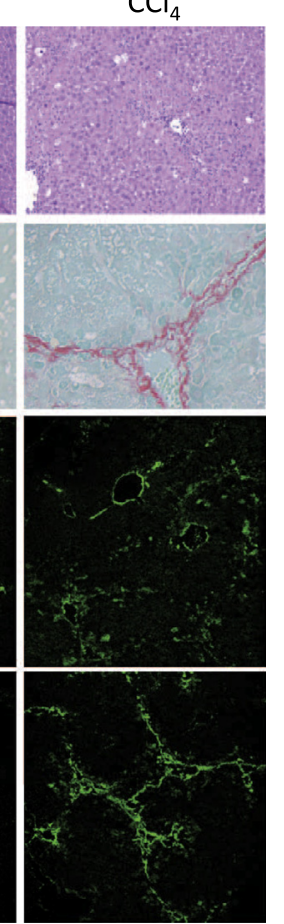

D

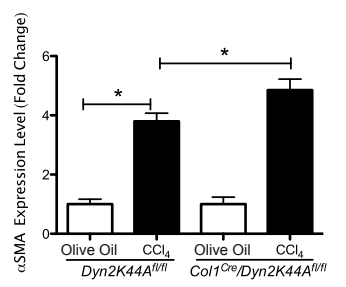

Figure $5 \mathrm{Col1}^{\text {Cre }} /$ Dyn2K44A $A^{f l f l}$ mice show enhanced liver fibrosis in response to $\mathrm{CCl}_{4}$-induced liver injury. Mice were subjected to $\mathrm{CCl}_{4}$ or olive oil for 6 weeks. Hematoxylin and eosin (H\&E) staining and Sirius Red staining were performed from paraffin liver sections. A: Immunofluorescence was performed on frozen liver sections using antibodies against $\alpha$-smooth muscle actin $(\alpha-S M A$; green) and fibronectin (FN; green). Representative images are shown. B: Fibrosis level was read and scored via H\&E and Sirius Red staining single blinded by a pathologist (T.M.). C-E: Collagen deposition, $\alpha$-SMA, and $\mathrm{FN}$ immunofluorescence were quantified using ImageJ, as detailed in Materials and Methods. $n=8$ (B-E). ${ }^{*} P<0.05$. Scale bars $=50 \mu \mathrm{m} . \mathrm{CCl}_{4}$, carbon tetrachloride.
E

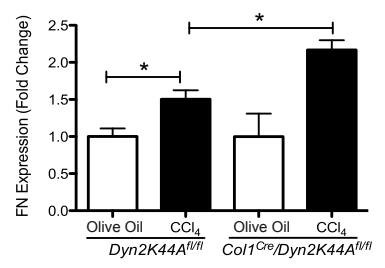

indicated a role for sphingosine-1-phosphate (S1P)/SK1 in HSC activation and liver fibrosis in vivo. ${ }^{13}$ This work demonstrated that liver fibrosis was attenuated by the sphingosine-1-phosphate receptor (S1PR)2 antagonist, further implicating a key role for S1P/SK1 in liver fibrosis, which was also required for HSC activation. ${ }^{13}$ Our current work shows SK1 mRNA levels were up-regulated in BDL and carbon tetrachloride models and significantly increased in $\mathrm{Coll}^{\mathrm{Cre}} / \mathrm{Dyn} 2 \mathrm{~K} 44 \mathrm{~A}^{\mathrm{fl} / \mathrm{fl}}$ compared to littermate controls (Figure 6, A and B). We next explored the relationship between Dyn2K44A and HSC activation in vitro using an adenoviral overexpression system. Dyn2 mRNA was upregulated similarly in Dyn2K44A cells or Dyn2WT cells (Figure 6C). However, SK1 mRNA and protein levels were markedly increased in Dyn2K44A, but not in LacZ or Dyn2WT (Figure 6, D and E). We also analyzed $\alpha$-SMA, FN, and ColI mRNA levels by real-time PCR, but observed no significant change in these HSC activation markers with Dyn2K44A overexpression (Supplemental Figure S1). We tested the effect of Dyn2K44A on SK1 in two other liver cell types, endothelial cells and hepatocytes; however, the
SK1 mRNA up-regulation in liver sinusoidal ECs or HepG2 was not notable (Figure 6, F and G). Thus, we show that increased SK1 by Dyn2K44A overexpression is a positive feedback loop selectively in HSCs, compared to other liver cell types. Furthermore, a complementary approach to the Dyn2K44A dominant negative construct, we knocked down Dyn2 using a siRNA and again found that SK1 mRNA was up-regulated (Figure 6, H and I). To examine whether SK1 up-regulation in Dyn2K44A HSCs was regulated at the transcriptional level, we performed luciferase-based reporter assays in HSCs. A construct containing a 1980-bp fragment amplified from the 5-flanking region of $S K 1$ gene was transfected into LacZ, Dyn2K44A, and Dyn2WT cotransfected HSCs. We observed that the promoter activity of the SK1 construct was threefold higher in Dyn2K44A than LacZ or WT (Figure 6J). These data indicated that disruption of Dyn2 activity increases SK1 expression by enhancing its transcription. Thus, these data supported a model in which Dyn2K44A increases HSC activation through upregulation of SK1. 

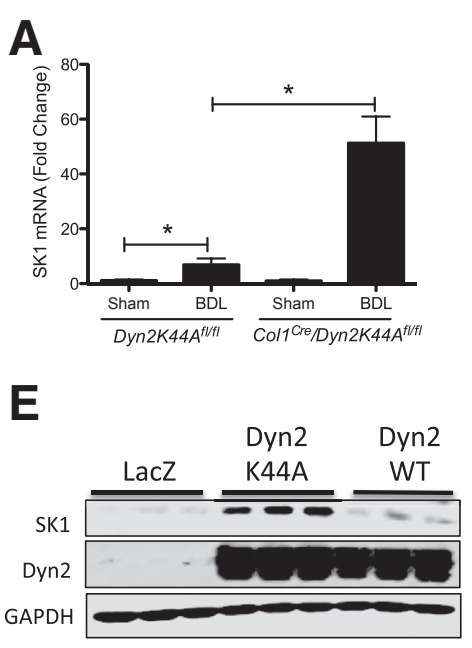

H

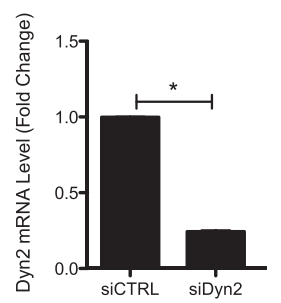

B
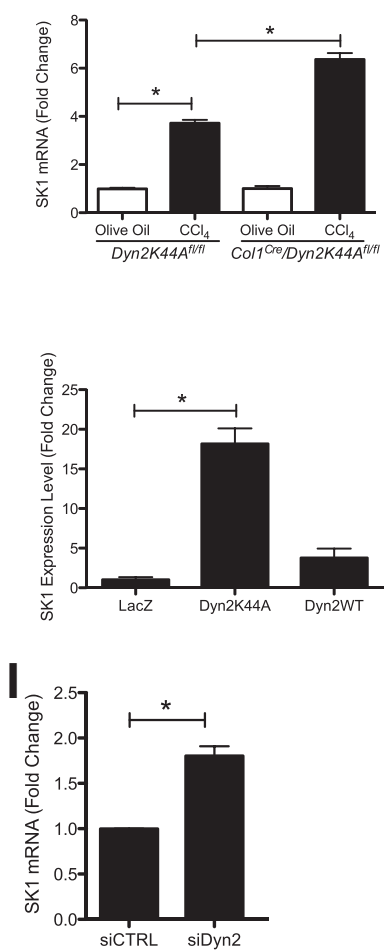

C

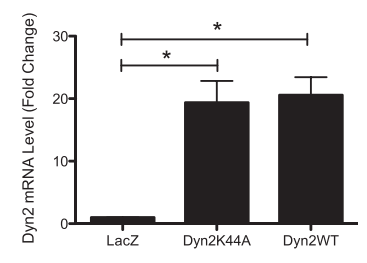

$\mathbf{F}$

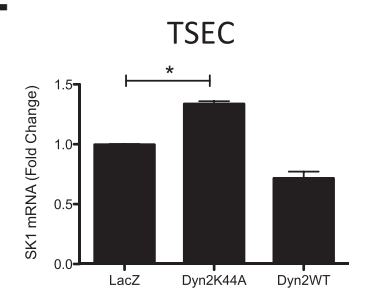

G

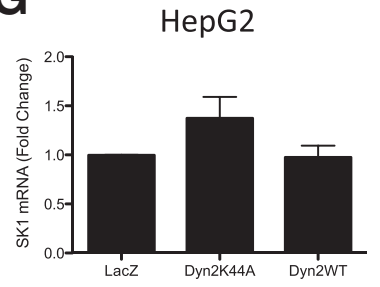

Figure 6 Dyn2K44A up-regulates sphingosine kinase-1 (SK1) expression in HSCs. A and B: Bile duct ligation (BDL) or CCl 4 model liver samples were subject to real-time PCR to examine SK1 mRNA levels. C: HSCs were infected with LacZ, Dyn2K44A, and Dyn2WT. The infection efficiency was tested using real-time PCR (no significant difference was observed between Dyn2K44A and Dyn2WT). D: mRNA from LacZ, Dyn2K44A, and Dyn2WT HSCs were subjected to real-time PCR for SK1 quantification. E: Lysates from LacZ, Dyn2K44A, and Dyn2WT HSCs were subjected to Western blot for SK1. Glyceraldehyde-3-phosphate dehydrogenase (GAPDH) serves as a loading control, and Dyn2 was a marker of infection efficiency. $\mathbf{F}$ and $\mathbf{G}$ : Liver sinusoidal ECs (TSECs) and HepG2 cells were infected with LacZ, Dyn2K44A, or Dyn2WT and then subjected to real-time PCR for SK1 (TSEC, significant change between groups; HepG2, no significant change between groups). H: HSCs were transfected with siCTRL or siDyn2. Transfection efficiency was tested by real-time PCR. I: siCTRL and siDyn HSCs were subjected to realtime PCR for SK1. J: HSCs were transfected with $400 \mathrm{ng}$ of a SK1 construct and $100 \mathrm{ng}$ of renilla, and infected with LacZ, Dyn2K44A, or Dyn2WT to examine the role of Dyn2 in SK1 transcriptional activation. The relative luciferase values were normalized to the renilla reading. $n=6(\mathbf{A}$ and $\mathbf{B}, \mathbf{B D L}) ; n=8(\mathbf{A}$ and $\mathbf{B}$, carbon tetrachloride); $n=12(\mathbf{C}$ and $\mathbf{D}) ; n=3(\mathbf{E}, \mathbf{H}$, and $\mathbf{I}) ; n=4(\mathbf{F}$ and $\mathbf{G}) .{ }^{*} P<0.05 . \mathrm{CCl}_{4}$, carbon tetrachloride; siCTRL, CTRL siRNA; siDyn2, Dyn2 siRNA.

\section{SK1 Up-Regulation Enhances AKT Signaling and Migration of HSCS}

We next sought to determine whether Dyn2K44A-induced up-regulation of SK1 leads to HSC migration because HSC migration is important for fibrogenesis. ${ }^{23}$ We have previously shown that SK1 mediates HSC activation through upregulation of AKT signaling and induction of HSC migration. ${ }^{24}$ Indeed, pAKT was up-regulated in Dyn2K44A HSCs compared to Dyn2WT (Figure 7A). To provide further evidence that SK1 was the primary mediator of Dyn2K44Ainduced AKT signaling in HSCs, we incubated Dyn2K44A, Dyn2WT, or LacZ infected HSCs with the SK1 inhibitor dimethylsphingosine, or the S1P antagonist, FTY720. Dimethylsphingosine and FTY720 both blocked AKT phosphorylation in Dyn2K44A HSCs (Figure 7B). These data provide evidence that Dyn2K44A induction of SK1 increases AKT signaling in HSCs.

We next sought to test whether conditioned media from Dyn2K44A cells can induce HSC migration. Transwell assays were performed in the presence of conditioned media derived from LacZ, Dyn2K44A, or Dyn2WT infected HSCs. Dyn2K44A HSC-derived conditioned media increased HSC migration 3.2-fold compared to conditioned media from LacZ or Dyn2WT HSC (Figure 7C). Inhibition of the SK1/S1P axis with the pharmacological inhibitors FTY720, dimethylsphingosine, or AKTviii significantly attenuated the Dyn2K44A conditioned media-induced HSC migration. These data indicate that disruption of Dyn2 in HSCs increases SK1 and S1P generation, which then contribute to HSC migration and fibrogenesis.

\section{Discussion}

Although the pathogenesis of liver fibrosis is not fully defined, the activation of HSCs is recognized as a sentinel step in its progress. ${ }^{25,26}$ In the current study, we investigate the effect of Dyn2 disruption on liver fibrosis by using a transgenic mouse model. This study contains the following novel findings: 


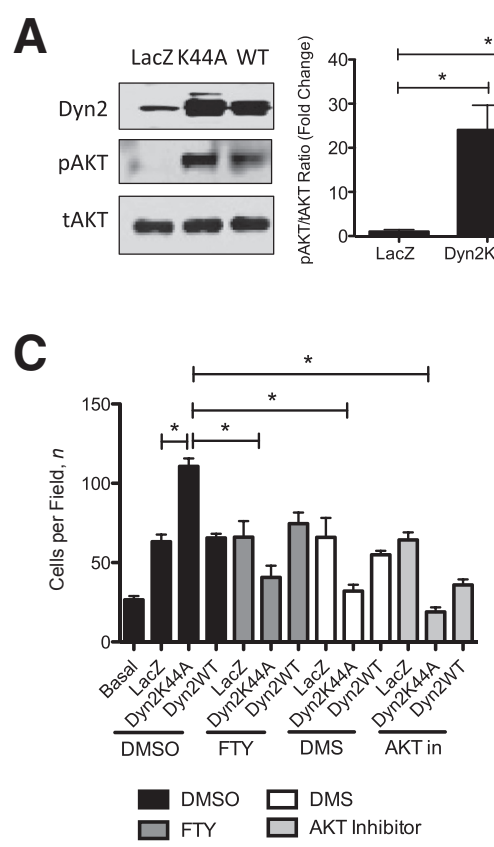

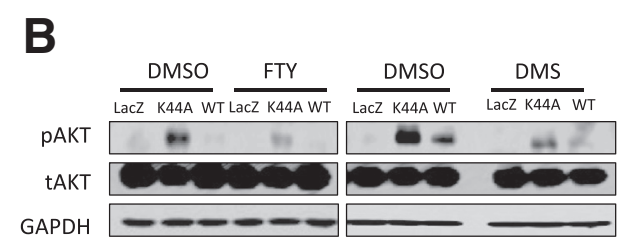

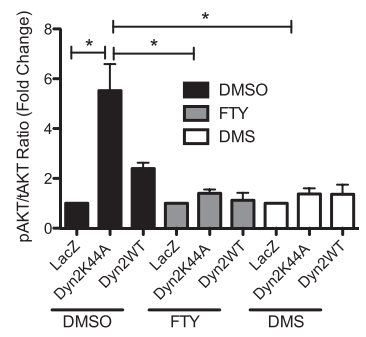

Figure 7 Dyn2K44A-induced sphingosine kinase-1 (SK1) up-regulation enhances AKT signaling and migration of HSCs. A: LacZ, Dyn2K44A, and Dyn2WT HSCs were subjected to Western blot (WB) for pAKT and tAKT. Dyn2 was used as a positive control for viral infection and overexpression levels. B: LacZ, Dyn2K44A, and Dyn2WT HSCs were treated with dimethyl sulfoxide (DMSO) as a control, or one of two SK1 inhibitors: FTY720 (10 nmol/L) or DMS $(10 \mathrm{nmol} / \mathrm{L})$. Cell lysates from different samples were subjected to WB for pAKT, tAKT, and glyceraldehyde-3-phosphate dehydrogenase as a loading control. Quantification was shown on the right. C: The effect of the conditioned media from LacZ, Dyn2K44A, or Dyn2WT HSCs on HSC migration was studied using transwell assay. The conditioned media or basal media were incubated with HSCs for 24 hours. To inhibit SK1/S1P axis or AKT, pharmacological inhibitor, DMS $(10 \mathrm{nmol} / \mathrm{L})$ and FTY720 $(10 \mathrm{nmol} / \mathrm{L})$, and AKT inhibitor viii $(25 \mathrm{nmol} / \mathrm{L})$ were added to the conditioned media before incubation with $\mathrm{HSC}$ and migration measurements. Quantification of data in terms of number of cell per field was shown. $n=4(\mathbf{A}-\mathbf{C}) .{ }^{*} P<0.05$. AKT, protein kinase B (PKB); DMS, dimethylsphingosine; FTY, FTY720; WT, wild-type.

i) disruption of Dyn2 in HSCs promotes fibrogenesis in vivo; ii) Dyn2K44A or siDyn2 in HSCs increases SK1 expression; and iii) Dyn2K44A HSCs increasing AKT signaling and migration is SK1 dependent. Given existing information implicating SK1 in liver fibrosis, we anticipate that this mechanism may contribute to HSC activation and fibrogenesis in response to disruption of Dyn2 function.

Dyn2 is a critical component of the endocytic machinery, known to regulate endocytosis of RTKs. ${ }^{10,27}$ We observed blocked TfR endocytosis in Dyn2K44A HSCs, confirming the loss of Dyn2 function in our system through the Dyn2K44A mutation. However, little is known about the relationship between Dyn2, endocytosis, and liver fibrosis. Prior studies using a mouse line that conferred Dyn2K44A expression selectively in endothelial cells impaired angiogenesis in vivo by disrupting RTK endocytosis and signaling. ${ }^{10}$ Because RTKs play an important role in liver fibrosis and HSC activation, we originally hypothesized that expression of Dyn2K44A in HSCs would impair fibrogenesis by decreasing HSC activation. To test our hypothesis, we crossed transgenic Dyn $2 K 44 A^{f l f l}$ mice with Coll $^{\text {Cre }}$ mice to generate mice with Dyn2K44A expressing in HSCs, selectively. Unexpectedly, in vivo data showed that expression of Dyn2K44A in HSCs did not ameliorate liver fibrogenesis, but instead, increased fibrosis.

We therefore explored the possible mechanisms mediating this effect. Dyn2 has varied functions in different cell types. Gong et $\mathrm{al}^{27}$ reported that inhibition of Dyn2 in hepatocellular carcinoma cells increased cell migration, colony formation, and invasion. In their study, Dyn2 knockdown reduced epidermal growth factor receptor internalization, which led to the activation of downstream Ras/extracellular signal-regulated kinase $1 / 2$ signaling. Lee et $\mathrm{al}^{28}$ reported that depletion of Dyn2 in cultured ECs impaired vascular endothelial growth factor receptor endocytosis and enhanced vascular endothelial growth factor signaling, as well as impaired integrin internalization. In HSCs, Dyn2K44A is reported to induce accumulation of RTKs on HSC surface, and enhanced RTK induced signaling; however, additional studies show that Dyn2K44A overexpression in HSCs blocks collagen internalization and matrix metalloproteinase production. ${ }^{29}$ In the present study in HSCs, the Dyn2K44A mechanism of action appeared through transcriptional regulation rather than through membrane receptor trafficking, as discussed below.

We observed transcriptional activation of SK1 by Dyn2K44A. This transcriptional mechanism of effect of Dyn2 is supported by previous publications. ${ }^{30,31} \mathrm{~A}$ recent study showed that Dyn2 can transcriptionally regulate p53 and hypoxia-inducible factors. ${ }^{30,31}$ Because the Dyn2 protein structure does not contain an obvious nucleus location sequence, ${ }^{32}$ the transcriptional effects of Dyn2 are likely to be indirect. For example, in a recent article, disruption of Dyn2 increased activator protein-1, a transcription factor that is also implicated in SK1 transcription. ${ }^{33,34}$ We found selective up-regulation of SK1 in HSCs that overexpress DynK44A, but not in hepatocytes or ECs. As we and others have shown induction of SK1 expression during HSC activation, we postulate that Dyn2K44A-mediated increases in SK1 serve as a positive feedback loop that is not present in other liver cell types. Further studies will be necessary to fully elucidate how Dyn2K44A regulates SK1 transcription in HSCs.

Based on our previous studies, S1P is an important phospholipid that regulates HSC migration, proliferation, angiogenesis, and differentiation. ${ }^{13} \mathrm{~S} 1 \mathrm{P}$ is generated by SK1 or 
SK2, which phosphorylate sphingosine to S1P. Previously, we showed that SK1 was up-regulated in carbon tetrachlorideand BDL-induced liver fibrosis, and exosomal SK1 was required for HSC migration, indicative of HSC activation. ${ }^{13}$ $\mathrm{S} 1 \mathrm{P}$ is a profibrogenic factor in the liver via its interaction with $\mathrm{S} 1 \mathrm{PR}_{2}$, which was demonstrated in $\mathrm{S}_{1} \mathrm{PR}_{2}$-deficient animals. ${ }^{35}$ Bone marrow-derived immune cells were also involved in the progression of liver fibrosis by secreting S1P to activate HSC via $\mathrm{S}_{\mathrm{PR}} .{ }^{36}$ Both animal models and human tissue showed up-regulation of SK1/S1P during liver fibrosis. ${ }^{37,38}$ Dyn2K44A induced SK1 up-regulation in HSCs, which contributes to the activation of HSCs, may provide a mechanism for our observations in vivo. Last, we showed that Dyn2K44A HSCs up-regulate AKT phosphorylation and increase HSC migration. Indeed, we were able to confirm this model because pharmacological inhibition of the SK1/S1P axis abolished AKT phosphorylation in Dyn2K44A cells, and conditioned media from these cells were unable to induce HSC migration. In summary, Dyn2K44A expression in HSCs up-regulates SK1 transcription and S1P generation and thereby contributes to HSC activation and liver fibrogenesis.

\section{Acknowledgments}

We thank David A. Brenner, M.D., and Tatiana Kisseleva, M.D., Ph.D., for provision of $\mathrm{Coll}^{\mathrm{Cre}}$ mice.

R.W. and V.H.S. developed the concept, designed experiments, and wrote the manuscript. R.W. and Q.D. performed animal work. T.M.D.A., S.C., and U.Y. provided technique support. M.T. helped with pathology analysis. C.D. helped with transwell assay. J.L.M. and R.C.H. helped with manuscript writing.

\section{Supplemental Data}

Supplemental material for this article can be found at http://dx.doi.org/10.1016/j.ajpath.2016.09.001.

\section{References}

1. Bataller R, Brenner DA: Liver fibrosis. J Clin Invest 2005, 115: 209-218

2. Friedman SL: Mechanisms of hepatic fibrogenesis. Gastroenterology 2008, 134:1655-1669

3. Friedman SL: Hepatic stellate cells: protean, multifunctional, and enigmatic cells of the liver. Physiol Rev 2008, 88:125-172

4. Ferguson SM, De Camilli P: Dynamin, a membrane-remodelling GTPase. Nat Rev Mol Cell Biol 2012, 13:75-88

5. Doherty GJ, McMahon HT: Mechanisms of endocytosis. Annu Rev Biochem 2009, 78:857-902

6. Luwor RB, Chin X, McGeachie AB, Robinson PJ, Zhu HJ: Dynamin II function is required for EGF-mediated Stat3 activation but not Erk1/2 phosphorylation. Growth Factors 2012, 30:220-229

7. Willinger T, Ferguson SM, Pereira JP, De Camilli P, Flavell RA: Dynamin 2-dependent endocytosis is required for sustained S1PR1 signaling. J Exp Med 2014, 211:685-700

8. Lee MY, Skoura A, Park EJ, Landskroner-Eiger S, Jozsef L, Luciano AK, Murata T, Pasula S, Dong Y, Bouaouina M,
Calderwood DA, Ferguson SM, De Camilli P, Sessa WC: Dynamin 2 regulation of integrin endocytosis, but not VEGF signaling, is crucial for developmental angiogenesis. Development 2014, 141: $1465-1472$

9. Damke H, Binns DD, Ueda H, Schmid SL, Baba T: Dynamin GTPase domain mutants block endocytic vesicle formation at morphologically distinct stages. Mol Biol Cell 2001, 12:2578-2589

10. Yaqoob U, Jagavelu K, Shergill U, de Assuncao T, Cao S, Shah VH: FGF21 promotes endothelial cell angiogenesis through a dynamin-2 and Rab5 dependent pathway. PLoS One 2014, 9:e98130

11. Wang Y, Gao J, Zhang D, Zhang J, Ma J, Jiang H: New insights into the antifibrotic effects of sorafenib on hepatic stellate cells and liver fibrosis. J Hepatol 2010, 53:132-144

12. Wang R, Ding Q, Yaqoob U, de Assuncao TM, Verma VK, Hirsova P, Cao S, Mukhopadhyay D, Huebert RC, Shah VH: Exosome adherence and internalization by hepatic stellate cells triggers sphingosine 1-phosphate-dependent migration. J Biol Chem 2015, 290:30684-30696

13. Kisseleva T, Cong M, Paik Y, Scholten D, Jiang C, Benner C, Iwaisako K, Moore-Morris T, Scott B, Tsukamoto H, Evans SM, Dillmann W, Glass CK, Brenner DA: Myofibroblasts revert to an inactive phenotype during regression of liver fibrosis. Proc Natl Acad Sci U S A 2012, 109:9448-9453

14. Papeleu P, Vanhaecke T, Henkens T, Elaut G, Vinken M, Snykers S, Rogiers V: Isolation of rat hepatocytes. Methods Mol Biol (Clifton, NJ) 2006, 320:229-237

15. Malhi H, Bronk SF, Werneburg NW, Gores GJ: Free fatty acids induce JNK-dependent hepatocyte lipoapoptosis. J Biol Chem 2006, 281: 12093-12101

16. Huebert RC, Jagavelu $\mathrm{K}$, Liebl AF, Huang BQ, Splinter PL, LaRusso NF, Urrutia RA, Shah VH: Immortalized liver endothelial cells: a cell culture model for studies of motility and angiogenesis. Lab Invest 2010, 90:1770-1781

17. Yang L, Kwon J, Popov Y, Gajdos GB, Ordog T, Brekken RA, Mukhopadhyay D, Schuppan D, Bi Y, Simonetto D, Shah VH Vascular endothelial growth factor promotes fibrosis resolution and repair in mice. Gastroenterology 2014, 146:1339-1350e1

18. Simonetto DA, Yang HY, Yin M, de Assuncao TM, Kwon JH, Hilscher M, Pan S, Yang L, Bi Y, Beyder A, Cao S, Simari RD, Ehman R, Kamath PS, Shah VH: Chronic passive venous congestion drives hepatic fibrogenesis via sinusoidal thrombosis and mechanical forces. Hepatology 2015, 61:648-659

19. Henley JR, Krueger EW, Oswald BJ, McNiven MA: Dynamin mediated internalization of caveolae. J Cell Biol 1998, 141:85-99

20. de Assuncao TM, Lomberk G, Cao S, Yaqoob U, Mathison A, Simonetto DA, Huebert RC, Urrutia RA, Shah VH: New role for Kruppel-like factor 14 as a transcriptional activator involved in the generation of signaling lipids. J Biol Chem 2014, 289:15798-15809

21. Greenhalgh SN, Conroy KP, Henderson NC: Cre-ativity in the liver: transgenic approaches to targeting hepatic non-parenchymal cells Hepatology 2015, 61:2091-2099

22. Bridle KR, Crawford DHG, Ramm GA: Identification and characterization of the hepatic stellate cell transferrin receptor. Am J Pathol 2010, 162:1661-1667

23. Semela D, Das A, Langer D, Kang N, Leof E, Shah V: Platelet-derived growth factor signaling through ephrin-b2 regulates hepatic vascular structure and function. Gastroenterology 2008, 135:671-679

24. Morales-Ruiz M, Cejudo-Martín P, Fernández-Varo G, Tugues S, Ros J, Angeli P, Rivera F, Arroyo V, Rodés J, Sessa WC, Jiménez W: Transduction of the liver with activated Akt normalizes portal pressure in cirrhotic rats. Gastroenterology 2003, 125:522-531

25. Hernandez-Gea V, Friedman SL: Pathogenesis of liver fibrosis. Annu Rev Pathol 2011, 6:425-456

26. Moreira RK: Hepatic stellate cells and liver fibrosis. Arch Pathol Lab Med 2007, 131:1728-1734

27. Gong C, Zhang J, Zhang L, Wang Y, Ma H, Wu W, Cui J, Wang Y, Ren Z: Dynamin2 downregulation delays EGFR endocytic trafficking 
and promotes EGFR signaling and invasion in hepatocellular carcinoma. Am J Cancer Res 2015, 5:702-713

28. Lee RT, Berditchevski F, Cheng GC, Hemler ME: Integrin-mediated collagen matrix reorganization by cultured human vascular smooth muscle cells. Circ Res 1995, 76:209-214

29. Bi Y, Mukhopadhyay D, Drinane M, Ji B, Li X, Cao S, Shah VH: Endocytosis of collagen by hepatic stellate cells regulates extracellular matrix dynamics. Am J Physiol Cell Physiol 2014, 307: C622-C633

30. Fish KN, Schmid SL, Damke H: Evidence that dynamin-2 functions as a signal-transducing GTPase. J Cell Biol 2000, 150:145-154

31. Joshi HP, Subramanian IV, Schnettler EK, Ghosh G, Rupaimoole R, Evans C, Saluja M, Jing Y, Cristina I, Roy S, Zeng Y, Shah VH, Sood AK, Ramakrishnan S: Dynamin 2 along with microRNA-199a reciprocally regulate hypoxia-inducible factors and ovarian cancer metastasis. Proc Natl Acad Sci U S A 2014, 111:5331-5336

32. Hinshaw JE: Dynamin and its role in membrane fission. Annu Rev Cell Dev Biol 2000, 16:483-519
33. Szymanska E, Skowronek A, Miaczynska M: Impaired dynamin 2 function leads to increased AP-1 transcriptional activity through the JNK/c-Jun pathway. Cell Signal 2016, 28:160-171

34. Huang K, Huang J, Chen C, Hao J, Wang S, Huang J, Liu P, Huang H: AP-1 regulates sphingosine kinase 1 expression in a positive feedback manner in glomerular mesangial cells exposed to high glucose. Cell Signal 2014, 26:629-638

35. Yang L, Yue S, Yang L, Liu X, Han Z, Zhang Y, Li L: Sphingosine kinase/sphingosine 1-phosphate (S1P)/S1P receptor axis is involved in liver fibrosis-associated angiogenesis. J Hepatol 2013, 59:114-123

36. Olivera A, Allende ML, Proia RL: Shaping the landscape: metabolic regulation of S1P gradients. Biochim Biophys Acta 2013, 1831:193-202

37. Alvarez SE, Milstien S, Spiegel S: Autocrine and paracrine roles of sphingosine-1-phosphate. Trends Endocrinol Metab 2007, 18:300-307

38. Li C, Zheng S, You H, Liu X, Lin M, Yang L, Li L: Sphingosine 1-phosphate (S1P)/S1P receptors are involved in human liver fibrosis by action on hepatic myofibroblasts motility. J Hepatol 2011, 54: 1205-1213 